\title{
Molecular Detection of Colistin Resistance Gene mcr-1 in Gram- negative Rods Isolated from Hospitalized Patients in Khartoum State
}

\author{
Areej Zakaria ${ }^{1 *}$, Mawada Rhmtallah ${ }^{1}$, Zeinab Mohammed Abdo ${ }^{1}$, MF Ismail ${ }^{2}$, Abdelhakam H. Ali $^{3}$, Mosab Nouraldein \\ Mohammed Hamad ${ }^{4}$ \\ ${ }^{1}$ Faculty of Medical Laboratory Science, Sudan International University Khartoum, Sudan \\ ${ }^{2}$ Faculty of Medical Laboratory Science, Sudan University of Science and Technology Khartoum, Sudan \\ ${ }^{3}$ Faculty of Medical Laboratory Science, University of Albutana, Sudan \\ ${ }^{4}$ Phylum of Medical Parasitology, Department of Medical Laboratory Sciences, Faculty of Health Science, Elsheikh Abdallah Elbadri University, \\ Berber, Sudan
}

DOI: $\underline{10.36348 / \text { sjpm.2020.v05i08.005 }}$ | Received: 04.08 .2020 | Accepted: 13.08 .2020 | Published: 16.08 .2020

*Corresponding author: Areej Zakaria

\section{Abstract}

Background: The emergence of the plasmid-mediated $m c r-1$ colistin-resistance genes in bacteria poses a potential threat for treatment of patients, especially when hospitalized. The pinpoints of this study were to search for the presence of $m c r-$ lgenein the thick of gram negative rods (Eschericea coli, Klebsiella spieces, Pseudomonas speices, Proteus speices, Aceinetobacter species, Serritia and Citrobacter species) isolates from clinical specimen using Polymerase Chain Reaction (PCR) technique. Methods: In this study, 185 nonduplicate Enterobacteriaceae isolates from clinical specimens were tested. Antimicrobial susceptibility test on 11 antibiotics was done by Kirby baur disk diffusion method. The presence of mcr-1 gene was investigated by PCR technique. Results: Out of 185 non duplicate Enterobacteriaceae, 6 isolates show positive mcr-1 gene including one Isolate (16.4\%) Pseudomonas aeurginosa from urine sample, and 5 $(83.3 \%)$ Klebsiella pneumonia isolated from two wound sample (40\%), one high vaginal swab sample (20\%), one blood sample (20\%) and one urine sample (20\%). Most of Isolates that harboring mcr-1 gene detected by PCR isolated from patients were used antibiotics for treatment $(n=4(66.6 \%))$. Conclusion: Colistin (Polymycin E) which used for treating Gram- negative bacterial infections should be mainly used for treating the severest infection to preserve their effectiveness and to avoid transferring of the gene from one bacterium to other by the different methods of genes transferring.

Keywords: Molecular detection, Colistin Resistance Gene mcr-1, Gram-negative Rods, Khartoum, Sudan.

Copyright @ 2020: This is an open-access article distributed under the terms of the Creative Commons Attribution license which permits unrestricted use, distribution, and reproduction in any medium for non-commercial use (NonCommercial, or CC-BY-NC) provided the original author and sources are credited.

\section{INTRODUCTION}

Antimicrobial resistance occur when microorganisms such as bacteria, viruses, fungi and parasites change in ways that render the medications used to cure the infections they cause ineffective [1]. When the microorganisms become resistant to most antimicrobials they are often referred to as "superbugs [1]". This is a major concern because a resistant infection may kill, can spread to others, and imposes huge costs to individuals and society [1]. Antimicrobial resistance (AMR), or drug resistance, develops when microbes no longer respond to a drug that previously treated them effectively [2]. Multidrug resistance (MDR) Gram-negative organisms for which no adequate therapeutic options exist, a joint initiative by the European Centre for Disease Prevention and Control (ECDC) and the Centers for Disease Control and Prevention (CDC) recently created a standardized international definitions for multidrug-resistant(MDR), extensively drug-resistant (XDR) and pandrug-resistant (PDR) with an aim to enhance the comparability of data and promote better comprehension of the problem of highly drug-resistant bacteria[3]. MDR was defined as acquired non-susceptibility to at least one agent in three or more antimicrobial categories, XDR was defined as non-susceptibility to at least one agent in all but two or fewer antimicrobial categories (i.e. bacterial isolates remain susceptible to only one or two categories) and PDR was defined as non-susceptibility to all agents in all antimicrobial categories [3].

Polymyxins, a group of polypeptide antibiotics that consists of 5 chemically different compounds (polymyxins A-E), were discovered in 1947 [4]. Only polymyxin $\mathrm{B}$ and polymyxin $\mathrm{E}$ (colistin) have been used in clinical practice [4]. The polymyxins are active 
against selected gram-negative bacteria, including Acinetobacter species, Pseudomonas aeruginosa, Klebsiella species, and Enterobacter species [5]. Polymyxins have been used extensively worldwide in topical optic and ophthalmic solutions [5]. Colistin is an old class of cationic, which act by disrupting the bacterial membranes resulting in cellular death [6]. Until recently, mechanisms of colistin resistance were limited to chromosomal mutations which confer a high fitness cost and cannot be transferred between organisms [7], the use of colistin has its own disadvantages because it is a neurotoxic and nephrotoxic agent, despite the toxicity of this relatively old agent, colistin is the last viable effective option for the treatment of invasive bloodstream infections that are due to carbapenemase-producing Gram negative rod [7]. Overuse of colistin has recently led to the emergence of resistance to this lifesaving agent [8]. The emergence of the first plasmid-mediated colistin resistance mechanism mcr-1, in Enterobacteriaceae was identified in China in late 2015 [4]. A novel plasmidmediated colistin resistance mechanism, encoded by the mcr-1 gene, has been identified, and has since been detected worldwide. The mcr-1colistin resistance mechanism is a major threat due to its lack of fitness cost and ability to be transferred between strains and species. Surveillance of colistin resistance mechanisms is critical to monitor the development and spread of resistance [7].

Gram-negative bacteria are facultative anaerobic and can be differentiated based on their ability to ferment glucose, produce nitrate, and produce catalase or oxidase [9]. This organisms cause infections including pneumonia, bloodstream infections, wound or surgical site infections, and meningitis in healthcare settings. Resistant to multiple drugs and are increasingly resistant to most available antibiotics. Add to that these bacteria have built-in abilities to find new ways to be resistant and can pass along genetic materials that allow other bacteria to become drugresistant as well. CDC's aggressive recommendations, if implemented, can prevent the spread of gramnegative rods resistance. Gram-negative infections include those caused by Klebsiella, Acinetobacter, Pseudomonas aeruginosa and E. coli, as well as many other less common bacteria [10]. Nosocomial infections caused by MDR and XDR Gram-negative pathogens represent a major threat worldwide [10]. Infections caused by resistant gram-negative bacteria are becoming increasingly prevalent and now constitute a serious threat to public health worldwide because they are difficult to treat and are associated with high morbidity and mortality rates [11]. The growing resistance among GNB to commonly used antibiotics has led to the resurgence of the use of antibiotics such as colistin as a last-resort treatment option [8]

\section{MATERIALS AND METHODS Bacterial isolates}

A total of 185 isolates of E. coli, Klebsiella species, Pseudomonas species, Proteus species, Aceintobactor and Citrobactor were detected from clinical samples. Urine 73 (39.46\%), swabs 46 (24.9\%), wound swabs $31(16.8 \%)$, blood 22 (11.9\%), sputum 7 $(3.8 \%)$, high vaginal swab $4(2.2 \%)$, tissue $1(.5 \%)$ and 1 aspirate $(.5 \%)$. These isolates were collected from the laboratories of Omer Sawi hospital and Military hospital from April to July 2019. This study was approved by the Institutional Review Board (IRB) and Ethics Committee of the Sudan International University. The isolates were transferred to the Medical Microbiology lab and were subcultured on MacConkey agar media for further checking and taking pure culture. After incubation of plates at $37^{\circ} \mathrm{C}$ for $18-24 \mathrm{hrs}$, the isolated colonies were identified by Gram staining and usual biochemical tests such as Kligler Iron agar, Siammon's citrate agar, Christensen urea agar, Semi solid Nutrient agar and amino acid tryptophan broth according to CLSI guidelines[12].

\section{Antimicrobial susceptibility testing (AST)}

AST was performed using disk diffusion method (Kirby-Bauer) on Mueller hinton agar plates following the Clinical and Laboratory Standards Institute guidelines [12]. The used antibiotic disks were colistin $(10 \mu \mathrm{g})$, ciprofloxacin $(5 \mu \mathrm{g})$, imipenem (10 $\mu \mathrm{g})$, ceftazidime $(30 \mu \mathrm{g})$, amikacin $(30 \mu \mathrm{g})$, amoxicillin $(10 \mu \mathrm{g})$, cefoxitin $(30 \mu \mathrm{g})$, gentamycin $(10 \mu \mathrm{g})$, cholroamphinicol $(30 \mu \mathrm{g})$, cefotaxime $(30 \mu \mathrm{g})$, and anti Pseudomonal (azetonam $(30 \mu \mathrm{g}), \quad$ pipracillin

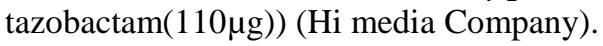

\section{DNA extraction and PCR amplification}

By using Guanidine extraction method we took 3-5 colonies from Isolate after purification and we put it in $400 \mu \mathrm{l}$ of $10 \%$ phosphate buffer saline, centrifuged for 5 minutes at 12000 RPM, discarded the supernatant and added $400 \mu 1$ of lysis buffer $+200 \mu 1$ of guanidine $+50 \mu \mathrm{l}$ of ammonium acetate $+5 \mu \mathrm{l}$ of proteinase $\mathrm{K}$ enzyme, Vortexed and incubated at $65^{\circ} \mathrm{C}$ for 2 hours after that add $400 \mu 1$ of cold coloroform, vortexed, centrifuged for 5 minutes at 12000 RPM and then we collected $400 \mu \mathrm{l}$ of upper layer in new eppendorff tube, we added $1 \mathrm{ml}$ of absolute ethanol, mixed and incubated overnight in $-20^{\circ} \mathrm{C}$, we brought to room temperature to 5 minutes, centrifuged for 5 minutes, discarded absolute ethanol, then added $400 \mu 1$ of $70 \%$ ethanol, centrifuged for 5 minutes at 6000 RPM, discarded $70 \%$ ethanol then we let it to dry on tissue for an hour and after that we added $40 \mu 1$ of distelled water for injection, vortexed for few seconds and stored at $-20^{\circ} \mathrm{C}[13]$. 
Detection of $m c r-1$ gene by polymerase chain reaction $(\mathrm{PCR})$

DNA amplification was performed using a thermocycler (Eppendorf, Hamburg, Germany).We added $5 \mu 1$ of master mix $+5 \mu 1$ of double distelled water $+.5 \mu \mathrm{l}$ of each primer (forward and reverse), and $4 \mu \mathrm{L}$ of extracted DNA as template. The DNA amplification was performed based on the following program: initial denaturation at $94^{\circ} \mathrm{C}$ for 5 minutes, 35 cycles of denaturation at $94^{\circ} \mathrm{C}$ for $1 \mathrm{~min}$, annealing at $52^{\circ} \mathrm{C}$ for a minute, extension at $72^{\circ} \mathrm{C}$ for a minute and a final extension at $72^{\circ} \mathrm{C}$ for 10 minutes. The PCR products were separated by electrophoresis on a $1.5 \%$ agarose gel containing $2 \mu \mathrm{l}$ of ethidium bromide. The bands were visualized under UV light using a gel documentation system.

Table-1: Shows Primer used for PCR of antimicrobial resistance mcr-1 gene

\begin{tabular}{|c|c|c|c|c|}
\hline Target gene & \multicolumn{2}{|c|}{ Primer sequence (5 to 3) } & Ampliconsize (bp) & Source ofreference \\
\hline mcr-1 & FR & $\begin{array}{c}\text { ATGATGCAGCATACTTCTGTG } \\
\text { TCAGCGGATGAATGCGGTG }\end{array}$ & 350 & $(20)$ \\
\hline
\end{tabular}

\section{STATISTICAL ANALYSIS}

It was done manually and Chi-square with a statistical significant $P$-value $<0.05$.

\section{RESULTS}

In this study, among 185 examined Enterobacteriaceae isolates, 53 isolates were identified as E. coli (28.6\%), 87 Isolates as K. pneumoniae (47\%),
21 Isolates as Pseudomonas (11.4\%), 17 Isolates as Proteus (9.2\%), 4 Isolates as Aceintobactor (2.2\%), one Isolate as Serritia (.6\%) and 1 Isolate as Citrobactor $(.6 \%)$. These isolates were collected from different clinical specimens and were screened for antibiotic resistance and presence of $m c r-1$ gene. The distributions of isolates in the specimens were as follows:

Table-2: Shows the distributions of isolates in the specimens

\begin{tabular}{|c|c|}
\hline Type of sample & No of Isolates (of 185 samples) \\
\hline Urine & $73(39.46 \%)$ \\
\hline Blood & $22(11.9 \%)$ \\
\hline Sputum & $7(3.8 \%)$ \\
\hline Wound & $31(16.8 \%)$ \\
\hline Swab & $46(24.9 \%)$ \\
\hline Tissue & $1(.5 \%)$ \\
\hline High vaginal swab & $4(2.2 \%)$ \\
\hline Aspirate & $1(.5 \%)$ \\
\hline
\end{tabular}

It is important to mention that most isolates were obtained from urine specimens. 6(3.24\%) isolates were positive to colistin mcr-1 gene by PCR including 1 (16.4\%) Pseudomonas from urine sample, child male and don't use antibiotics and sensitive by AST and 5 $(83.3 \%) \quad$ Klebsiella pneumoniae were detected as follow:

Table-3: Shows the Full discretions of Positive mcr-1 gene Isolates by PCR technique

\begin{tabular}{|l|l|l|l|l|l|}
\hline The isolates & Type of sample & $\begin{array}{l}\text { Age of } \\
\text { patient }\end{array}$ & $\begin{array}{l}\text { Gender of } \\
\text { patient }\end{array}$ & $\begin{array}{l}\text { Using of } \\
\text { antibiotic }\end{array}$ & $\begin{array}{l}\text { Susceptibility } \\
\text { by AST }\end{array}$ \\
\hline Pseudomonas aeuroginosa & Urine & 8 years & Male & No & Susceptible \\
\hline Klebsiella pneumonia & Wound & 67 years & Male & Yes & Resistant \\
\hline Klebsiella pneumonia & Urine & 35 years & Female & Yes & Resistant \\
\hline Klebsiella pneumonia & Wound & 12 years & Male & Yes & Resistant \\
\hline Klebsiella pneumonia & High vaginal swab & 65 years & Female & Yes & Susceptible \\
\hline Klebsiella pneumonia & Blood & 3 days & Female & No & Resistant \\
\hline
\end{tabular}


Picture.1. PCR product (309 pb) run on ethidum bromide gel visualized under UV light:

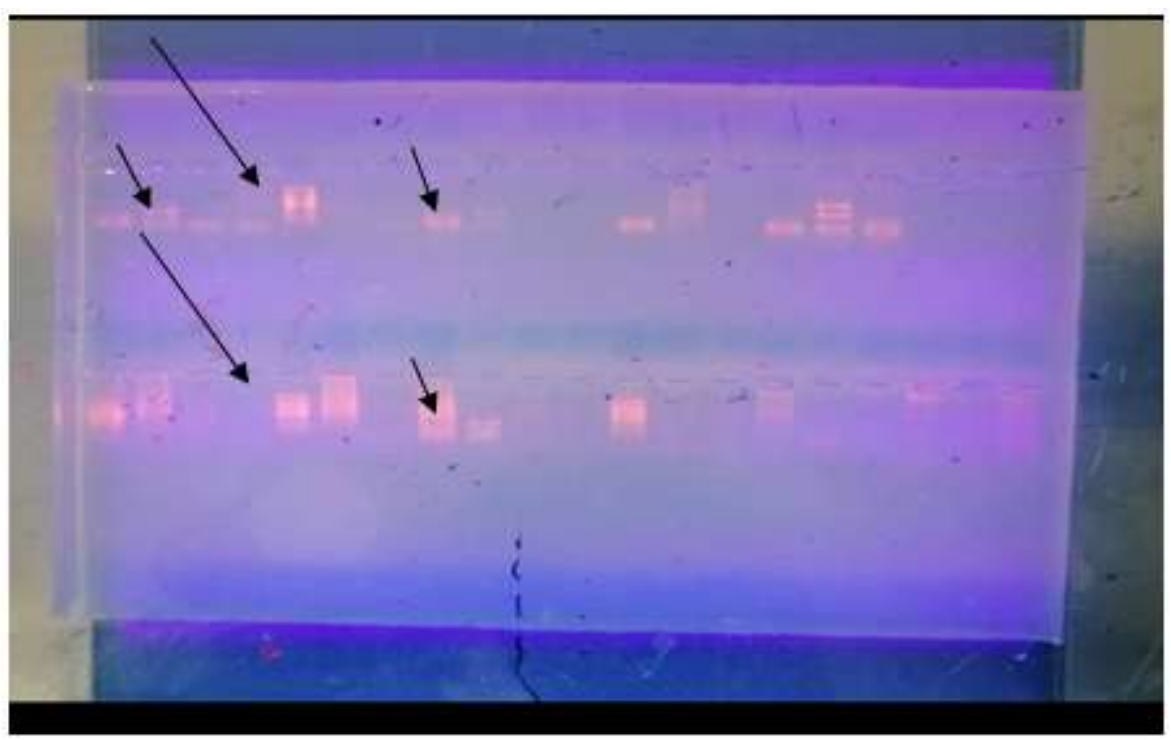

Short arrow: represent band of mcr-1 gene (309 bp).

Long arrow: represent the ladder

Most of Isolates that harboring mcr-1 gene detected by PCR the patients were under antibiotics therapy $(4(66.6 \%))$. About 4 of $52(7.7 \%)$ of resistant Isolates by AST were positive by PCR and 2 of 133 (1.5\%) of sensitive Isolate by AST were positive by PCR.

Table-4: Shows the relationship between the presence of mcr-1 gene (by PCR) and its expression (by AST)

\begin{tabular}{|c|c|c|c|c|}
\hline & Isolates by AST & Sensitive Isolates by AST & Column total & P. value \\
\hline Positive by PCR & $011(4)$ & $012(2)$ & 5 & \multirow[t]{3}{*}{0.05} \\
\hline Negative by PCR & $021(48)$ & $022(131)$ & 179 & \\
\hline Raw total & 52 & 133 & Grand total $=185$ & \\
\hline
\end{tabular}

H0: the presence of resistant to colistin by AST independant on the presence of mor-1 gene which is detected by PCR. H1: the presence of resistant to colistin by AST dependant on the presence of mor-1 gene which is detected by PCR.

Degree of freedom $($ d.f $)=($ raws -1$) \times($ columns -1$)$ $=(2-1) \times(2-1)=1 \times 1=1$

$\mathrm{P}$ value $=0.05$

Tabulated $\chi 2$ with $P$ value $.05=3.84$

$2=\Sigma(\mathrm{o}-\mathrm{E}) 2 \div \mathrm{E} \chi$

O: represent observed value

E: represent expected value

E: represent raw total $\mathrm{x}$ column total $\div$ grand total $\chi 2=5.57$

Since the calculated $\chi 2$ (5.57) is greater than tabulated one (3.84), we reject the null hypothesis, and we conclude that the presence of resistant to colistin by AST dependant on the presence of mor-1 gene which is detected by PCR.

\section{DISCUSSION}

The spread of antibiotics resistance to a wide range of antibiotics such as beta-lactams, aminoglycosides and carbapenems is a global challenge to the health systems. Using colistin is regarded as the last resort for treating infections caused by MDR-gram negative rods, especially Enterobacteriaceae[7]. However, its nephrotoxicity and neurotoxicity impacts have reduced its application as a routine prescribed drug [14].

Elisabeth and her colleges they found that $2 /$ 576 patients were positive to mcr-1 $(0.35 \%)$ [15]. Lin Cao and his group they realized that six $(0.06 \%)$ strains carried the mcr-1 gene from 1112 isolated E. coli [16]. Their results differ from ours cause that; they collected higher number of samplesthan we, focused in stool sample, E.coli speicifically and finally study area.

Elisabetta et al they found that $25(8.3 \%)$ harbored the gene (mcr-1) and this gene is spread to many gram negative bacteria ( $K$. pneumonia, $K$. oxytoca, E. coli, Acenitobactor lwoffii, Enterobactor cloacae, Enterobactor agglomerans, Citrobactor ferundii, Pseudomonas aeruginosa and Pseudomonas putida [17]. Jun Lu et al. worked out to find the prevalence of mcr-1 gene in Salmonella typhimurium and they found that 3 of 62 samples $(4.8 \%)$ were positive to mcr-1 gene and all of these three were susceptible to colistin [18]. The differs between our 
results and theirs due to dissimilarity in type of samples, organisms, sample size and study area.

\section{CONCLUSION}

Propagating of Enterobacteriaceae strains harboring $m c r-1$ containing plasmids should fail the colistin-included therapy regimen which is used as a last line of treatment against MDR gram-negative bacterial infections.

\section{RECOMMENDATIONS}

Increasing supervision of colistin-resistance mechanisms for monitoring their accession and spread is vital. Persevering efforts to ensure the discreet use of colistin (and indeed all antibiotics) both in agriculture and in health-care systems are welcome.

Continuous monitoring is very important for determining the exact frequency of $m c r-1$ gene among gram-negative bacteria in human. Reevaluation of polymyxins application in human and implementation of large regular screening of human isolates for $m c r-1$ gene should be an important step in preventing the spread of this gene to the human isolates.

\section{REFERENCES}

1. World Health Organization. (2017). Online Question and Answer.

2. James, M. (2018). Antibiotic resistance: What you need to know, Medical News Today.

3. Magiorakos, A. P., Srinivasan, A., Carey, R. B., Carmeli, Y., Falagas, M. E., Giske, C. G., ... \& Paterson, D. L. (2012). Multidrug-resistant, extensively drug-resistant and pandrug-resistant bacteria: an international expert proposal for interim standard definitions for acquired resistance. Clinical microbiology and infection, 18(3), 268-281.

4. Yi-Yun. L., Yang. W., Timothy. R.W., Ling-Xian. Y., Rong. Z., James. S. (2015). Emergance of plasmid-mediated colistin mechanism MCR-1 in animal and human beings in China, the lancet, 16(2);161- 168.

5. Falagas, M. E., Kasiakou, S. K., \& Saravolatz, L. D. (2005). Colistin: the revival of polymyxins for the management of multidrug-resistant gramnegative bacterial infections. Clinical infectious diseases, 40(9), 1333-1341.

6. Bialvaei, A. Z., \& Samadi Kafil, H. (2015). Colistin, mechanisms and prevalence of resistance. Current medical research and opinion, 31(4), 707-721.

7. Newton-Foot, M., Snyman, Y., Maloba, M. R. B., \& Whitelaw, A. C. (2017). Plasmid-mediated mcr1 colistin resistance in Escherichia coli and Klebsiella spp. clinical isolates from the Western
Cape region of South Africa. Antimicrobial Resistance \& Infection Control, 6(1), 1-7.

8. Garbati, M. A., Abdulhak, A. B., Baba, K., \& Sakkijha, H. (2013). Infection due to colistinresistant Enterobacteriacae in critically-ill patients. The Journal of Infection in Developing Countries, 7(10), 713-719.

9. Wanger, A., Chavez, V., Huang, R., Wahed, A., Dasgupta, A., \& Actor, J. K. (2017). Microbiology and molecular diagnosis in pathology: a comprehensive review for board preparation, certification and clinical practice. Elsevier.

10. Barbier, F., Andremont, A., Wolff, M., \& Bouadma, L. (2013). Hospital-acquired pneumonia and ventilator-associated pneumonia: recent advances in epidemiology and management. Current opinion in pulmonary medicine, 19(3), 216-228.

11. Kaye, K. S., \& Pogue, J. M. (2015). Infections caused by resistant gram- negative bacteria: epidemiology and management. Pharmacotherapy: The Journal of Human Pharmacology and Drug Therapy, 35(10), 949-962.

12. Waites, K. B., Bade, D. J., Bébéar, C., Brown, S. D., Davidson, M. K., Duffy, L. B., ... \& Totten, P. A. (2011). Methods for antimicrobial susceptibility testing for human mycoplasmas; approved guideline.

13. Tan, S. C., \& Yiap, B. C. (2009). DNA, RNA, and protein extraction: the past and the present. Journal of Biomedicine and Biotechnology, 2009.

14. Falagas, M. E., Rafailidis, P. I., \& Matthaiou, D. K. (2010). Resistance to polymyxins: mechanisms, frequency and treatment options. Drug Resistance Updates, 13(4-5), 132-138.

15. Terveer, E. M., Nijhuis, R. H., Crobach, M. J., Knetsch, C. W., Veldkamp, K. E., Gooskens, J., ... \& Claas, E. C. (2017). Prevalence of colistin resistance gene (mcr-1) containing Enterobacteriaceae in feces of patients attending a tertiary care hospital and detection of a mcr-1 containing, colistin susceptible E. coli. PLoS One, 12(6), e0178598.

16. Cao, L., Li, X., Xu, Y., \& Shen, J. (2018). Prevalence and molecular characteristics of mcr-1 colistin resistance in Escherichia coli: isolates of clinical infection from a Chinese University Hospital. Infection and drug resistance, 11, 1597.

17. Caselli, E., D’Accolti, M., Soffritti, I., Piffanelli, M., \& Mazzacane, S. (2018). Spread of mcr-1Driven Colistin Resistance on Hospital Surfaces, Italy. Emerging infectious diseases, 24(9), 1752.

18. Lu, J., Quan, J., Zhao, D., Wang, Y., Yu, Y., \& Zhu, J. (2019). Prevalence and molecular characteristics of mcr-1 gene in Salmonella typhimurium in a tertiary hospital of Zhejiang Province. Infection and drug resistance, 12, 105. 